\title{
Gold(I)-Catalyzed Formation of Bicyclo[4.2.0]oct-1-enes
}

\author{
Ryan J. Felix ${ }^{\dagger}$, Osvaldo Gutierrez ${ }^{\ddagger}$, Dean J. Tantilloł,", and Michel R. Gagné ${ }^{\dagger,{ }^{*}}$ \\ tDepartment of Chemistry, University of North Carolina at Chapel Hill, Chapel Hill, North Carolina \\ 27599-3290, United States \\ ‡Department of Chemistry, University of California—Davis, Davis, California 95616, United States
}

\begin{abstract}

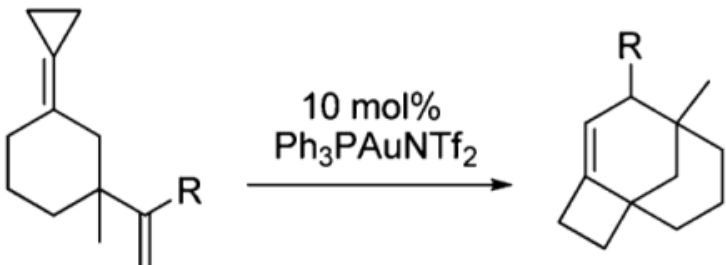

Gold(I) catalysts effectively promote the Cope rearrangement of acyclic 1,5-dienes bearing a terminal cyclopropylidene. When this methodology is applied to cyclic substrates an unexpected transformation occurs, resulting in the formation of a tricyclic compound incorporating a bicyclo[4.2.0]oct-1-ene core, a portion of which is found in a number of natural products. Density functional theory calculations (M06 and M06-2X) reveal insight into the mechanism and thermodynamics of this unique transformation.
\end{abstract}

Transition-metal catalysts play a prominent role in promoting the kinetic viability of a variety of synthetic reactions. When used in combination with thermodynamically favorable transformations, like the consumption of $\mathrm{C}-\mathrm{C}$ unsaturation ${ }^{1,2}$ and the release of ring strain, ${ }^{3-11}$ numerous useful methodologies have emerged. The construction of complex polycyclic structures from relatively simple starting materials has been the subject of a number of research efforts aimed at accessing biomimetic carbocycles. ${ }^{12-16}$

In our own effort to utilize ring strain and unsaturation for biomimetic polycyclization reactions, we discovered that 1,5-dienes with a terminal alkylidene cyclopropane preferentially undergo Cope rearrangements instead of the expected cascade cyclizations under $\mathrm{Au}(\mathrm{I})$ catalysis (Scheme 1) ${ }^{17}$ This reaction is driven thermodynamically by the relief of ring strain in the cyclopropylidene moiety. ${ }^{18,19}$ In this paper, we extend the scope of this rearrangement to cyclic substrates like $\mathbf{1}(\mathrm{R}=\mathrm{Me}, \mathrm{Ph})$ with the goal of using alkylidene cyclopropane strain release to access medium sized carbocycles, ${ }^{20-22}$ which suffer from their own strain (Scheme 1). To our surprise, the Cope rearrangement was seemingly not followed, and tricyclic compounds 2 , which feature a bicyclo[4.2.0] oct-1-ene core, are

\footnotetext{
(c) 2013 American Chemical Society

*Corresponding Author: mgagne@unc.edu, djtantillo@ucdavis.edu.

The authors declare no competing financial interest.

Supporting Information

${ }^{1} \mathrm{H}$ and ${ }^{13} \mathrm{C}$ NMR spectroscopic data for all new compounds and discussion of 2D NMR spectroscopic data for compound $\mathbf{2 b}$, as well as coordinates and energies for all computed structures and complete ref 34 . This material is available free of charge via the Internet at http://pubs.acs.org.
} 
formed instead (eq 1). This bicyclic skeleton is present in a number of natural products including welwitindolinone $\mathrm{A}$, and the protoilludane class of sesquiterpenes. ${ }^{23-26}$<smiles>[R]C(=C)C1(I)CCCC(=C2CC2)C1</smiles>

$1 \mathrm{a} R=\mathrm{Me}$ $1 \mathrm{~b} R=\mathrm{Ph}$<smiles>C=C[C@]1(C)C(C#N)=C2C(CC1Cl)C(C)(C)C1(C(=O)Nc3ccccc31)C2C</smiles>

Welwitindolinone A
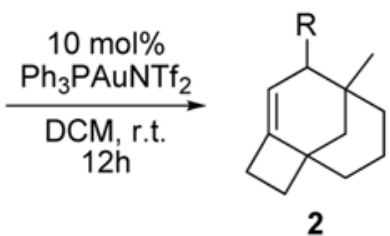

2a $\mathrm{R}=\mathrm{Me} 76 \%$

2b R $=\mathrm{Ph} 88 \%$

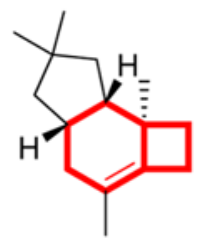

$(-)-\Delta^{6}-$ Protoilludene

Employing 10 mol \% of the Gagosz catalyst, ${ }^{27} \mathrm{Ph}_{3} \mathrm{PAuNTf}_{2}$ (Tf $=$

trifluoromethanesulfonyl) allows the reaction to proceed cleanly over $12 \mathrm{~h}$ at room temperature to a single product (2). The rearranged $\mathbf{2 a}$ and $\mathbf{2 b}$ are isolable via flash chromatography in good yield ( $76 \%$ and $88 \%$ yield, respectively) and were identified by an analysis of the NOESY NMR spectra of $\mathbf{2} \mathbf{b}$. The assignment of $\mathbf{2 a}$ was made based on similarities in the NMR spectra to $\mathbf{2 b}$. Key correlations are shown in Figure 1.

The phenyl ring was assigned to the concave face of the molecule through a correlation between $\mathrm{H}_{\mathrm{A}}(7.35 \mathrm{ppm})$ and $\mathrm{H}_{\mathrm{B}}(1.85 \mathrm{ppm})$, but not between $\mathrm{H}_{\mathrm{A}}$ and $\mathrm{H}_{\mathrm{C}}(1.50 \mathrm{ppm})$ (Figure 1a), as well as the signal between $H_{D}(3.40 \mathrm{ppm})$ and $\mathrm{H}_{\mathrm{E}}(1.75 \mathrm{ppm})$ (Figure 1b). The cisring configuration was assigned by the key interactions between the diaxially oriented $\mathrm{H}_{\mathrm{E}}$ (1.75 ppm) and $\mathrm{H}_{\mathrm{F}}(1.85 \mathrm{ppm}), \mathrm{H}_{\mathrm{G}}(1.90 \mathrm{ppm})$ with $\mathrm{H}_{\mathrm{I}}(3.0 \mathrm{ppm})$ (Figure 1c), and the supporting resonance between $\mathrm{H}_{\mathrm{A}}$ and $\mathrm{H}_{\mathrm{B}}$. A more in-depth discussion of the NOESY data is provided in the Supporting Information.

To explore the scope of the reaction a series of additional substrates were synthesized, varying in ring size and substitution on the pendant alkene (Scheme 2). These were obtained in two steps from the cyclic enone via a Cu-mediated Michael addition at low temperature, followed by a Wittig reaction. The former reactions were generally high yielding for commercially available Grignard reagents (68-94\%), but low yields were obtained from the Grignard reagent prepared from $a$-bromostyrene (18-58\%). The resulting ketone could be transformed, in poor to moderate yield, into the desired substrate with cyclopropyltriphenylphosphonium bromide and $\mathrm{NaH}$, facilitated by the phase transfer catalyst tris[2-(2-methoxyethoxy)ethyl]amine (TDA-1). ${ }^{33}$ It was hoped that the cyclopentylidene and cycloheptylidene substrates would provide access to tricyclic compounds that varied in ring structure (eqs 2 and 3, respectively). 
Unfortunately, the additional substrates gave neither the tricyclic products nor the analogous originally hypothesized medium ring products. Despite considerable experimentation, compounds 1c-e and $\mathbf{4 a}$ gave complex, intractable mixtures of compounds. Attempts to optimize via choice of solvent (nitromethane, 1,2-dichloroethane) and catalyst (e.g., $(R)$ $\operatorname{BINAP}(\mathrm{AuCl})_{2},(S)$-xylyl-PHANEPHOS $\left.(\mathrm{AuCl})_{2}\right)$, or activating agent $\left(\mathrm{AgBF}_{4}, \mathrm{AgSbF}_{6}\right.$, $\mathrm{AgPF}_{6}$ ) failed to simplify the reaction mixtures. It was observed that in the case of $\mathbf{4 a}$ the identity of the counterion affected the ratio of products observed in the GC-MS but not the number of species formed. For compounds $\mathbf{3 a}$ and $\mathbf{3} \mathbf{b}$ two major products were formed along with a number of minor products. Control reactions with $\mathrm{HCl} \cdot \mathrm{Et}_{2} \mathrm{O}$ gave the same products in the same ratios, as did a separate control reaction between $\mathbf{3 a}$ and $\mathrm{AgPF}_{6}$ in DCM, suggesting unproductive, Brønsted acid catalyzed alkene isomerization pathways. These isomerization reactions removed the ring strain of the methylenecyclopropane by transformation of the exocyclic alkene into an endocyclic olefin (within the cyclopentyl ring, see the Supporting Information). Identical control reactions with the 6- and 7-membered ring substrate analogues were sluggish and did not produce the same reaction mixtures observed as when $\mathrm{Au}(\mathrm{I})$ complexes were reacted. No tricyclic product was formed when the control reactions were run with $\mathbf{1 a}$ and $\mathbf{1 b}$, supporting a gold-catalyzed transformation for the production of 2 .
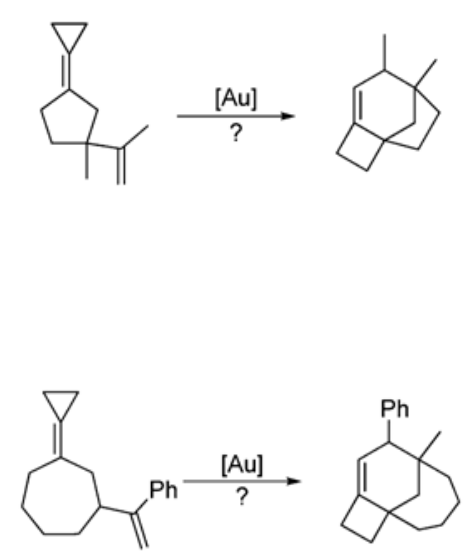

Insight into the reaction's mechanism and limitations was achieved through density functional theory (DFT) calculations using the M06/6-31G(d) method with the SDD basis set for Au and a DCE solvent continuum (CPCM), as implemented in GAUSSIAN09. ${ }^{34-37}$ Two potential pathways for the rearrangement were examined, the first proceeding through the originally envisioned Cope rearrangement/ring expansion, which initiates by $\mathrm{Au}(\mathrm{I})$ activation of the alkylidene cyclopropane (A, Figure 2). Cyclization initiated by complexation at this point of unsaturation is predicted to proceed with a barrier of $22 \mathrm{kcal} /$ mol (relative to $\mathbf{B}$ ), passing through tertiary carbocation $\mathbf{A 1}$ to give the cyclooctenes $\mathbf{A 2} / \mathbf{A 3}$ in a stepwise formal [3s,3s] sigmatropic rearrangement that is exergonic by $\sim 3-5 \mathrm{kcal} / \mathrm{mol}$ (see the Supporting Information for additional details). From this point, a second electrophilic cyclization is initated at the endocyclic alkene to give A4 (via A3-TS). ${ }^{38}$ Unexpectedly, no transition-state structure could be found to enable the direct ring expansion of the cyclopropane in A4. Instead, a conformational change is needed (via A4TS) to orient the bonds and associated orbitals for facile ring expansion (see A5). Expansion of the cyclopropane to the tetracyclic 6-5-4-3 intermediate C proceeds through A5-TS, the highest energy transition-state structure in the predicted pathway. Deprotonation followed 
by protodemetalation provides the complexed product $\mathbf{2 a}$, with the overall process being exergonic by more than $25 \mathrm{kcal} / \mathrm{mol}$.

An alternative pathway, which avoids formation of a cyclooctene intermediate but converges with pathway $A$ at structure $\mathbf{A 4}$, involves initiation of the Cope rearrangement at the pendant alkene instead of the cyclopropylidene moiety. In this pathway, cyclization of $\mathbf{B}$ to $\mathbf{A 4}$ proceeds directly through a transition-state structure (B-TS, $22.9 \mathrm{kcal} / \mathrm{mol}$ ) that is only slightly higher energy than A-TS.

The failure of substrates 1c-e was also probed computationally, and the results are summarized in Table 1. First, each of these reactions was found to be at least slightly endergonic, with each pathway suffering some additional deficiency. Substitution at $R_{2}$ creates steric interactions with the cyclohexyl ring and increases the relative energy of ATS. In these cases $((Z)-\mathbf{1 c}$ and -1e), a transition-state structure leading from the starting material to the ring-expanded product could not be located. In addition, compound $(E)-\mathbf{1 c}$, rearranges through a secondary carbocation rather than a tertiary carbocation (cf. Figure 2). While substrate 1d demonstrated an achievable ring-expansion barrier, the analogous A5-TS was too high in energy ( $31 \mathrm{kcal} / \mathrm{mol}$ ) for conversion to $\mathbf{C}$. The source of this high barrier appears to result from a steric clash between methyl groups.

In summary, we have described a $\mathrm{Au}(\mathrm{I})$-catalyzed Cope rearrangement of cyclic cyclopropylidenes into unique tricyclic compounds with a bicyclo[4.2.0]oct-1-ene core, a structural motif present in several classes of natural products. The reaction proceeds eficiently at room temperature to provide the products in good yields. Quantum chemical calculations provide insight into the mechanism and thermodynamics of the reaction.

\section{EXPERIMENTAL SECTION}

All reagents were purchased from commercial sources and used as received unless otherwise noted. All glassware was flame-dried under vacuum unless otherwise indicated. Anhydrous $\mathrm{CH}_{2} \mathrm{Cl}_{2}$, diethyl ether, and pentanes were passed through a column of alumina. Column chromatography was performed using SilaFlash P60 40-63 $\mu \mathrm{m}$ (230-400 mesh). All NMR spectra were recorded on either a 600 or $400 \mathrm{MHz}$ spectrometer at STP. ${ }^{1} \mathrm{H}$ and ${ }^{13} \mathrm{C}$ chemical shifts are reported in parts per million ( $\mathrm{ppm}$ ) relative to residual solvent resonances $\left(\mathrm{CDCl}_{3}\right.$ or $\left.\mathrm{CD}_{2} \mathrm{Cl}_{2}\right)$. High-resolution mass spectra (EI/HRMS) were obtained on a doublefocusing magnetic sector spectrometer.

\section{General Procedure A for Michael Addition Reactions (for Preparation of 3-Methyl-3- (prop-1-en-2-yl)cyclohexanone)}

To a flame-dried $100 \mathrm{~mL}$ round-bottom flask under $\mathrm{N}_{2}$ was added CuI $(3.45 \mathrm{~g}, 18.2 \mathrm{mmol}$, 2.00 equiv) and then THF $(18 \mathrm{~mL})$. The reaction vessel was cooled to $-41^{\circ} \mathrm{C}$ before the addition of the Grignard reagent, isopropenylmagnesium bromide ( $0.5 \mathrm{M} \mathrm{THF}, 36.3 \mathrm{~mL}$, $18.2 \mathrm{mmol}, 2.00$ equiv), over $30 \mathrm{~min}$. The reaction was stirred at $-41{ }^{\circ} \mathrm{C}$ for $30 \mathrm{~min}$ before the addition of 3-methyl-2-cyclohexen-1-one (1.03 mL, $9.08 \mathrm{mmol}, 1.00$ equiv) dissolved in THF $(9 \mathrm{~mL})$ via cannula. The reaction was then stirred $1.5 \mathrm{~h}$ at $-41^{\circ} \mathrm{C}$ before being quenched with saturated aqueous $\mathrm{NH}_{4} \mathrm{Cl}(50 \mathrm{~mL})$. The aqueous layer was separated, and the organic layer was washed two additional times with saturated aqueous $\mathrm{NH}_{4} \mathrm{Cl}$. The combined aqueous washes were then extracted with $\mathrm{Et}_{2} \mathrm{O}(2 \times)$. The combined organic layers were washed with brine until the aqueous layer was no longer blue-tinted. The organic layers were then dried over $\mathrm{MgSO}_{4}$, filtered, and concentrated in vacuo. Purification by silica gel chromatography (15\% EtOAc/petroleum ether) provided the product compound as a yellow oil ( $1.30 \mathrm{~g}, 94 \%$ yield $)$. 


\section{General Procedure B for Wittig cyclopropylidination (for Preparation of 3- Cyclopropylidene-1-methyl-1-(prop-1-en-2-yl)cyclohexane (1a))}

To a Schlenk flask loaded with a suspension of dry NaH $(0.102 \mathrm{~g}, 4.26 \mathrm{mmol}, 1.30$ equiv $)$ in THF ( $25 \mathrm{~mL}$ ) under $\mathrm{N}_{2}$ atmosphere was added cyclopropyltriphenylphosphonium bromide $(1.63 \mathrm{~g}, 4.26 \mathrm{mmol}, 1.30$ equiv) at rt. The reaction flask was then equipped with a condenser and heated to $62{ }^{\circ} \mathrm{C}$ for $18 \mathrm{~h}$. To the resulting orange suspension were then added the ketone (3-methyl-3-(prop-1-en-2-yl)cyclohexanone, $0.500 \mathrm{~g}, 3.28 \mathrm{mmol}, 1.00$ equiv) and tris[2-(2methoxyethoxy)ethyl]amine (TDA-1) $(0.105 \mathrm{~mL}, 0.328 \mathrm{mmol}, 0.10$ equiv) in THF (6 mL). The reaction was stirred for $5 \mathrm{~h}$ at $62{ }^{\circ} \mathrm{C}$ before being cooled to $\mathrm{rt}$ and quenched with saturated aqueous $\mathrm{NaHCO}_{3}$. The reaction was diluted with deionized $\mathrm{H}_{2} \mathrm{O}$ and $\mathrm{Et}_{2} \mathrm{O}$ before the layers were separated. The aqueous layer was extracted with $\mathrm{Et}_{2} \mathrm{O}(2 \times)$, and the combined organic layers were then washed with brine $(2 \times)$. The organic layer was then dried over $\mathrm{MgSO}_{4}$, filtered, and concentrated in vacuo. Purification by silica gel chromatography (hexanes) provided the product compound as a colorless oil (0.269 g, 47\%). A small amount of $\mathrm{CH}_{2} \mathrm{Cl}_{2}$ was used to load the material onto the column.

\section{General Procedure $\mathbf{C}$ for Preparation and Use of (1-Phenylvinyl)magnesium Bromide (Preparation of 3-Methyl-3-(1-phenylvinyl)cyclohexanone)}

To a flame-dried 100-mL three-neck RBF equipped with a condenser under $\mathrm{N}_{2}$ atmosphere were added $\mathrm{Mg}(0.467 \mathrm{~g}, 19.2 \mathrm{mmol}, 2.12$ equiv) and THF $(32.3 \mathrm{~mL})$ and a few small crystals of $\mathrm{I}_{2} . \boldsymbol{\alpha}$-Bromostyrene ( $2.35 \mathrm{~mL}, 18.1 \mathrm{mmol}, 2.00$ equiv) was dissolved in THF (4 $\mathrm{mL}$ ) and then added to the reaction mixture. The solution was heated to $70{ }^{\circ} \mathrm{C}$ for $5-15 \mathrm{~min}$ until the consumption of $\mathrm{Mg}$ appeared to have stopped. After being cooled to room temperature, the Grignard solution $(\sim 0.5 \mathrm{M})$ was transferred via cannula to a suspension of $\mathrm{CuI}\left(3.45 \mathrm{~g}, 18.1 \mathrm{mmol}, 1.00\right.$ equiv) in THF $(36 \mathrm{~mL})$ at $-41{ }^{\circ} \mathrm{C}$. The reaction was stirred at $-41{ }^{\circ} \mathrm{C}$ for $30 \mathrm{~min}$ before the addition of a solution of 3-methyl-2-cyclohexen-1-one (1.03 $\mathrm{mL}, 9.07 \mathrm{mmol}, 1.00$ equiv) dissolved in THF $(9 \mathrm{~mL})$ via cannula. The reaction was then stirred $1.5 \mathrm{~h}$ at $-41{ }^{\circ} \mathrm{C}$ before being quenched with saturated aqueous $\mathrm{NH}_{4} \mathrm{Cl}(50 \mathrm{~mL})$. The aqueous layer was separated, and the organic layer was washed two additional times with saturated aqueous $\mathrm{NH}_{4} \mathrm{Cl}$. The combined aqueous washes were then extracted with EtOAc $(2 \times)$. The combined organic layers were washed with brine until the aqueous layer was no longer blue-tinted and finally dried over $\mathrm{MgSO}_{4}$, filtered, and concentrated in vacuo. Purification by silica gel chromatography (15\% EtOAc/petroleum ether) provided the product compound as a yellow oil as a mixture with styrene ( $1.32 \mathrm{~g}$ total, $0.971 \mathrm{~g}$ product, $50 \%$ yield).

\section{General Procedure D for Au(I)-Catalyzed Rearrangement (for Preparation of 6,7- Dimethyltricyclo[5.3.1.0]undec-4-ene (2a))}

To a 1-dram vial equipped with a stirbar was added $\mathrm{Ph}_{3} \mathrm{PAuNTf}_{2}(0.011 \mathrm{~g}, 0.0142 \mathrm{mmol}$, 0.10 equiv) followed by DCM $(0.5 \mathrm{~mL})$. The reaction was stirred briefly before addition of 1a $(0.025 \mathrm{~g}, 0.142 \mathrm{mmol}, 1.00$ equiv). The reaction was then stirred for $12 \mathrm{~h}$ before being concentrated in vacuo. A pipet column was then used for purification by silica gel chromatography (hexanes) to provide the product compound as a colorless oil $(0.019 \mathrm{~g}$, $76 \%$ ). A small amount of DCM was used to add the material to the column.

3-Methyl-3-(prop-1-en-2-yl)cyclohexanone-Yellow oil (1.30 g, 94\% yield) ${ }^{1} \mathrm{H}$ NMR $\left(600 \mathrm{MHz}, \mathrm{CDCl}_{3}\right): \delta 4.80(\mathrm{~s}, 1 \mathrm{H}), 4.71(\mathrm{~s}, 1 \mathrm{H}), 2.58(\mathrm{~d}, J=14.4 \mathrm{~Hz}, 1 \mathrm{H}), 2.28(\mathrm{dt}, J=15.0$, $5.7 \mathrm{~Hz}, 1 \mathrm{H}), 2.22-2.18(\mathrm{~m}, 1 \mathrm{H}), 2.17(\mathrm{~d}, J=14.4 \mathrm{~Hz}, 1 \mathrm{H}), 1.91-1.87(\mathrm{~m}, 1 \mathrm{H}), 1.84-1.77(\mathrm{~m}$, $1 \mathrm{H}), 1.71-1.67(\mathrm{~m}, 1 \mathrm{H}), 1.69(\mathrm{~s}, 3 \mathrm{H}), 1.56(\mathrm{ddd}, J=13.2,9.6,3.6 \mathrm{~Hz}, 1 \mathrm{H}), 1.06(\mathrm{~s}, 3 \mathrm{H}) ;{ }^{13} \mathrm{C}$ NMR (150 MHz, $\left.\mathrm{CDCl}_{3}\right): \delta 211.9,150.0,112.0,52.7,43.9,41.0,35.0,27.0,22.0,19.3$. HRMS (EI+): calcd for $\mathrm{C}_{10} \mathrm{H}_{16} \mathrm{O}$ 152.12012, found 152.12093. 
3-Cyclopropylidene-1-methyl-1-(prop-1-en-2-yl)cyclohexane (1a)-Colorless oil $\left(0.269 \mathrm{~g}, 47 \%\right.$ yield). ${ }^{1} \mathrm{H}$ NMR $\left(600 \mathrm{MHz}, \mathrm{CDCl}_{3}\right): \delta 4.74(\mathrm{~s}, 1 \mathrm{H}), 4.73(\mathrm{~s}, 1 \mathrm{H}), 2.41(\mathrm{~d}, J=$ $13.2 \mathrm{~Hz}, 1 \mathrm{H}), 2.26-2.22(\mathrm{~m}, 1 \mathrm{H}), 2.17-2.14(\mathrm{~m}, 1 \mathrm{H}), 2.13(\mathrm{~d}, J=13.2 \mathrm{~Hz}, 1 \mathrm{H}), 1.71(\mathrm{~s}, 3 \mathrm{H})$, 1.71-1.68 (m, 1H), 1.55-1.50 (m, 2H), 1.47-1.44 (m, 1H), 0.99-0.96 (m, 4H), 0.94 (s, $3 \mathrm{H}) ;{ }^{13} \mathrm{C}$ NMR $\left(150 \mathrm{MHz}, \mathrm{CDCl}_{3}\right): \delta 152.9,126.1,113.9,109.4,43.8,41.0,36.5,33.2$, 25.9, 23.4, 19.8, 2.13, 2.10. HRMS (EI+): calcd for $\mathrm{C}_{13} \mathrm{H}_{20}$ 176.15650, found 176.15722.

3-Methyl-3-(1-phenylvinyl)cyclohexanone-Characterization data matched that previously reported. ${ }^{39}$

(1-(3-Cyclopropylidene-1-methylcyclohexyl)vinyl)benzene (1b)-Synthesized following general procedure B using 3-methyl-3-(1-phenyvinyl)cyclohexanone. Stirred $5 \mathrm{~h}$ at $62{ }^{\circ} \mathrm{C}$ after addition of ketone in THF. Purified by silica gel chromatography (hexanes) to give a colorless oil $\left(0.281 \mathrm{~g}, 36 \%\right.$ yield). ${ }^{1} \mathrm{H}$ NMR $\left(400 \mathrm{MHz}, \mathrm{CD}_{2} \mathrm{Cl}_{2}\right): \delta 7.30-7.22(\mathrm{~m}, 3 \mathrm{H})$, $7.16-7.13(\mathrm{~m}, 2 \mathrm{H}), 5.23(\mathrm{~d}, J=1.6 \mathrm{~Hz}, 1 \mathrm{H}), 4.82(\mathrm{~d}, J=1.6 \mathrm{~Hz}, 1 \mathrm{H}), 2.46(\mathrm{~d}, J=13.2 \mathrm{~Hz}$, $1 \mathrm{H}), 2.31(\mathrm{dt}, J=13.2,5.2 \mathrm{~Hz}, 1 \mathrm{H}), 2.23(\mathrm{~d}, J=12.8 \mathrm{~Hz}, 1 \mathrm{H}), 2.15-2.09(\mathrm{~m}, 1 \mathrm{H}), 1.75-1.69$ $(\mathrm{m}, 1 \mathrm{H}), 1.68-1.60(\mathrm{~m}, 1 \mathrm{H}), 1.59-1.46(\mathrm{~m}, 2 \mathrm{H}), 1.06(\mathrm{~s}, 3 \mathrm{H}), 1.02-0.89(\mathrm{~m}, 4 \mathrm{H}) ;{ }^{13} \mathrm{C}$ NMR $\left(100 \mathrm{MHz}, \mathrm{CD}_{2} \mathrm{Cl}_{2}\right.$ ): $\delta 159.6,144.0,129.7,127.8,126.8,126.1,114.5,113.5,44.5,41.5$, 37.2, 33.4, 25.9, 23.7, 2.21. HRMS (EI+): calcd for $\mathrm{C}_{18} \mathrm{H}_{22} 238.17215$, found 238.17139.

3-Methyl-3-(prop-1-enyl)cyclohexanone-Synthesized following general procedure A using 1-propenylmagnesium bromide solution ( $0.5 \mathrm{M}$ THF). Purified by silica gel chromatography (15\% EtOAc/petroleum ether) to give a yellow oil as a 1:1 inseperable mixture of $E / Z$ isomers $(0.981 \mathrm{~g}, 71 \%$ yield). Spectroscopic data reported is of the mixture of isomers. ${ }^{1} \mathrm{H}$ NMR $\left(600 \mathrm{MHz}, \mathrm{CD}_{2} \mathrm{Cl}_{2}\right)$ : $\delta 5.39$ (ddt $\left.J=12.0,7.8,7.2 \mathrm{~Hz}, 1 \mathrm{H}\right), 5.36-5.29$ $(\mathrm{m}, 2 \mathrm{H}), 5.20(\mathrm{dq}, J=11.7,1.8 \mathrm{~Hz}, 1 \mathrm{H}), 2.45(\mathrm{~d}, J=13.2 \mathrm{~Hz}, 1 \mathrm{H}), 2.34(\mathrm{dt}, J=13.8,1.8 \mathrm{~Hz}$, $1 \mathrm{H}), 2.22-2.16(\mathrm{~m}, 5 \mathrm{H}), 2.10(\mathrm{~d}, J=13.8 \mathrm{~Hz}, 1 \mathrm{H}), 1.95-1.91(\mathrm{~m}, 1 \mathrm{H}), 1.88-1.76(\mathrm{~m}, 4 \mathrm{H})$, 1.69 (dd, $J=7.2,1.8 \mathrm{~Hz}, 3 \mathrm{H}), 1.64-1.55(\mathrm{~m}, 3 \mathrm{H}), 1.62$ (d, $J=4.8 \mathrm{~Hz}, 3 \mathrm{H}), 1.16(\mathrm{~s}, 3 \mathrm{H})$, 1.00 (s, 3H). ${ }^{13} \mathrm{C}$ NMR (150 MHz, $\left.\mathrm{CD}_{2} \mathrm{Cl}_{2}\right): \delta 211.6,211.5,139.4,137.0,125.6,123.4$, 55.1, 52.8, 41.7, 41.4, 41.3, 38.1, 37.5, 28.2, 27.6, 23.1, 22.7, 18.4, 14.8. HRMS (EI+): calcd for $\mathrm{C}_{10} \mathrm{H}_{16} \mathrm{O}$ 152.12012, found 152.12048.

3-Cyclopropylidene-1-methyl-1-(prop-1-enyl)cyclohexane (1c)-Synthesized following general procedure B using 3-methyl-3-(prop-1-enyl)cyclohexanone. Stirred $2.5 \mathrm{~h}$ at $62{ }^{\circ} \mathrm{C}$ after addition of ketone in THF. Purified by silica gel chromatography (hexanes) to give a colorless oil as a 1:1 inseparable mixture of $E / Z$ isomers $(0.185 \mathrm{~g}, 32 \%$ yield $)$. Spectroscopic data reported is of the mixture of isomers. ${ }^{1} \mathrm{H} \mathrm{NMR}\left(600 \mathrm{MHz}, \mathrm{CD}_{2} \mathrm{Cl}_{2}\right): \delta$ $5.43-5.36(\mathrm{~m}, 1 \mathrm{H}), 5.35-5.29(\mathrm{~m}, 2 \mathrm{H}), 5.23(\mathrm{dq}, J=12.0,1.8 \mathrm{~Hz}, 1 \mathrm{H}), 2.39(\mathrm{~d}, J=12.6 \mathrm{~Hz}$, 1H), $2.29-2.23(\mathrm{~m}, 1 \mathrm{H}), 2.21(\mathrm{~d}, J=13.2 \mathrm{~Hz}, 1 \mathrm{H}), 2.17-2.10(\mathrm{~m}, 2 \mathrm{H}), 2.06(\mathrm{~d}, J=19.5 \mathrm{~Hz}$, $1 \mathrm{H}), 2.04(\mathrm{~d}, J=19.8,1 \mathrm{H}), 1.86-1.82(\mathrm{~m}, 1 \mathrm{H}), 1.71(\mathrm{dd}, J=7.2,1.8 \mathrm{~Hz}, 3 \mathrm{H}), 1.63(\mathrm{dd}, J=$ $4.8,1.2 \mathrm{~Hz}, 3 \mathrm{H}), 1.61-1.46(\mathrm{~m}, 6 \mathrm{H}), 1.44-1.38(\mathrm{~m}, 2 \mathrm{H}), 1.11(\mathrm{~s}, 3 \mathrm{H}), 1.03-0.95(\mathrm{~m}, 8 \mathrm{H})$, $0.91(\mathrm{~s}, 3 \mathrm{H}) .{ }^{13} \mathrm{C} \mathrm{NMR}\left(150 \mathrm{MHz}, \mathrm{CD}_{2} \mathrm{Cl}_{2}\right): \delta 141.9,139.3,126.4,126.2,123.9,121.1$, 114.2, 114.0, 47.7, 45.4, 39.3, 39.0, 38.9, 38.3, 33.5, 33.4, 27.2, 26.5, 24.2, 23.7, 18.6, 15.0, 2.21, 2.17, 2.11. HRMS (EI+): calcd for $\mathrm{C}_{13} \mathrm{H}_{20} 176.15650$, found 176.15742 .

(E)-3-(But-2-en-2-yl)-3-methylcyclohexanone-Synthesized following general procedure A using 1-methyl-1-propenylmagnesium bromide solution (0.5 M THF). Stirred for $2 \mathrm{~h}$ at $-41^{\circ} \mathrm{C}$ after addition of enone. Purified by silica gel chromatography (15\% EtOAc/petroleum ether) to give a yellow oil; $3: 1$ ratio of inseparable diastereomers $(1.13 \mathrm{~g}$, $75 \%$ yield). Spectroscopic data reported is of the mixture of isomers. ${ }^{1} \mathrm{H} \mathrm{NMR}(600 \mathrm{MHz}$, $\left.\mathrm{CD}_{2} \mathrm{Cl}_{2}\right): \delta 5.31(\mathrm{q}, J=7.8 \mathrm{~Hz}, 1 \mathrm{H}), 5.24(\mathrm{q}, J=6.6 \mathrm{~Hz},(1 / 3)=1 \mathrm{H}$, minor isomer), $2.69(\mathrm{~d}$, $J=13.8 \mathrm{~Hz}, 1 \mathrm{H}), 2.54(\mathrm{~d}, J=14.4 \mathrm{~Hz},(1 / 3)=1 \mathrm{H}$, minor isomer), $2.29-2.20(\mathrm{~m}, 2 \mathrm{H}), 2.18-$ 
$2.12(\mathrm{~m}, 2 \mathrm{H}), 1.93-1.74(\mathrm{~m}, 2 \mathrm{H}), 1.70-1.67(\mathrm{~m}, 6 \mathrm{H}), 1.56-1.55(\mathrm{~m}, 1 \mathrm{H}), 1.14(\mathrm{~s}, 3 \mathrm{H}), 1.03$ (s, $(1)=3 \mathrm{H}$, minor isomer). ${ }^{13} \mathrm{C} \mathrm{NMR}\left(150 \mathrm{MHz}, \mathrm{CD}_{2} \mathrm{Cl}_{2}\right): \delta 211.9,211.8,140.9,140.8$, 121.7, 119.4, 54.1, 53.0, 44.7, 44.3, 41.4, 41.3, 36.3, 35.3, 27.2, 26.2, 24.2, 22.8, 22.4, 15.9, 14.0, 12.4. HRMS (EI+): calcd for $\mathrm{C}_{11} \mathrm{H}_{18} \mathrm{O} 166.13577$, found 166.13636 .

(E)-1-(But-2-en-2-yl)-3-cyclopropylidene-1-methylcyclohexane (1d)—Synthesized following general procedure B using (E)-3-(but-2-en-2-yl)-3-methylcyclohexanone. Stirred $4.5 \mathrm{~h}$ at $62{ }^{\circ} \mathrm{C}$ after addition of ketone in THF. Purified by silica gel chromatography (hexanes) to give a colorless oil as an inseparable 3:1 ratio of diastereomers $(0.106 \mathrm{~g}, 17 \%$ yield). Spectroscopic data reported is of the mixture of isomers. ${ }^{1} \mathrm{H} \mathrm{NMR}(600 \mathrm{MHz}$, $\left.\mathrm{CD}_{2} \mathrm{Cl}_{2}\right): \delta 5.33-5.30(\mathrm{~m},(1 / 3)=1 \mathrm{H}$, minor isomer), 5.26-5.23 $(\mathrm{m}, 1 \mathrm{H}), 2.54(\mathrm{~d}, J=13.2$ $\mathrm{Hz}, 1 \mathrm{H}), 2.37(\mathrm{~d}, J=12.6 \mathrm{~Hz},(1 / 3)=1 \mathrm{H}$, minor isomer), 2.28-2.24 (m, $1 \mathrm{H}), 2.20-2.16(\mathrm{~m}$, $1 \mathrm{H}), 2.13(\mathrm{~d}, J=13.2 \mathrm{~Hz}, 1 \mathrm{H}), 2.01-1.97(\mathrm{~m}, 1 \mathrm{H}), 1.69(\mathrm{bs}, 6 \mathrm{H}), 1.60-1.50(\mathrm{~m}, 3 \mathrm{H}), 1.06(\mathrm{~s}$, $3 \mathrm{H}), 0.99$ (bs, $4 \mathrm{H}), 0.91$ (s, $(1)=3 \mathrm{H}$, minor isomer). ${ }^{13} \mathrm{C} \mathrm{NMR}\left(150 \mathrm{MHz}, \mathrm{CD}_{2} \mathrm{Cl}_{2}\right): \delta$ 143.23, 143.16, 126.9, 126.7, 120.3, 116.7, 114.1, 113.8, 45.4, 44.1, 41.64, 41.58, 37.6, $36.8,33.6,33.5,25.5,25.3,24.03,23.99,23.7,16.1,14.0,12.6,2.4,2.21,2.19,2.1$. HRMS (EI+): calcd for $\mathrm{C}_{14} \mathrm{H}_{22} 190.17215$, found 190.17239 .

3-Methyl-3-(2-methylprop-1-enyl)cyclohexanone-Synthesized following general procedure A using 2-methyl-1-propenylmagnesium bromide solution (0.5 M THF). Stirred $1.5 \mathrm{~h}$ at $-41{ }^{\circ} \mathrm{C}$ after addition of enone. Purified by silica gel chromatography (15\% EtOAc/ petroleum ether) to give a yellow oil (1.02 g, 68\% yield). ${ }^{1} \mathrm{H}$ NMR $\left(600 \mathrm{MHz}, \mathrm{CD}_{2} \mathrm{Cl}_{2}\right): \delta$ $5.03(\mathrm{~s}, 1 \mathrm{H}), 2.41(\mathrm{~d}, J=12.6 \mathrm{~Hz}, 1 \mathrm{H}), 2.21(\mathrm{~d}, J=4.8 \mathrm{~Hz}, 2 \mathrm{H}), 2.17(\mathrm{~d}, J=13.2 \mathrm{~Hz}, 1 \mathrm{H})$, 1.93 (bs, $1 \mathrm{H}), 1.83$ (bs, 2H), 1.71 (s, 3H), 1.67 (s, 3H), 1.60 (bs, $1 \mathrm{H}), 1.15$ (s, 3H). ${ }^{13} \mathrm{C}$ NMR $\left(150 \mathrm{MHz}, \mathrm{CD}_{2} \mathrm{Cl}_{2}\right.$ ): $\delta 211.7,133.6,131.0,55.6,41.4,40.9,38.3,28.3,27.6,23.1,19.5$. HRMS (EI+): calcd for $\mathrm{C}_{11} \mathrm{H}_{18} \mathrm{O} 166.13577$, found 166.13609 .

\section{3-Cyclopropylidene-1-methyl-1-(2-methylprop-1-enyl)-cyclohexane (1e)-}

Synthesized following general procedure B using 3-methyl-3-(2-methylprop-1enyl)cyclohexanone. Stirred $3 \mathrm{~h}$ at $62{ }^{\circ} \mathrm{C}$ after addition of ketone in THF. Purified by silica gel chromatography (hexanes) to give a colorless oil ( $0.206 \mathrm{~g}, 33 \%$ yield). ${ }^{1} \mathrm{H}$ NMR (600 $\left.\mathrm{MHz}, \mathrm{CD}_{2} \mathrm{Cl}_{2}\right): \delta 5.04(\mathrm{~s}, 1 \mathrm{H}), 2.35(\mathrm{~d}, J=13.2 \mathrm{~Hz}, 1 \mathrm{H}), 2.26-2.24(\mathrm{~m}, 1 \mathrm{H}), 2.12(\mathrm{bs}, 1 \mathrm{H})$, $2.02(\mathrm{~d}, J=12.6 \mathrm{~Hz}, 1 \mathrm{H}) 1.82-1.79(\mathrm{~m}, 1 \mathrm{H}), 1.70(\mathrm{~s}, 3 \mathrm{H}), 1.64(\mathrm{~s}, 3 \mathrm{H}) 1.57-1.54(\mathrm{~m}, 1 \mathrm{H})$, 1.51-1.45 (m, 1H), 1.42-1.38 (m, 1H), $1.08(\mathrm{~s}, 3 \mathrm{H}), 1.03-0.98(\mathrm{~m}, 4 \mathrm{H}) ;{ }^{13} \mathrm{C}$ NMR $(150$ $\left.\mathrm{MHz}, \mathrm{CD}_{2} \mathrm{Cl}_{2}\right): \delta 133.5,131.4,126.6,114.1,48.0,39.5,38.0,33.5,28.3,27.1,24.2,19.5$, 2.2, 2.1. HRMS (EI+): calcd for $\mathrm{C}_{14} \mathrm{H}_{22} 190.17215$, found 190.17146 .

3-Methyl-3-(prop-1-en-2-yl)cyclopentanone-Synthesized following general procedure A using isopropenylmagnesium bromide (0.5 M THF) and 3-methylcyclopent-2enone. Stirred $1.5 \mathrm{~h}$ at $-41^{\circ} \mathrm{C}$ after addition of enone. This material was sufficiently pure to be taken on without further purification as a yellow oil $\left(0.991 \mathrm{~g}, 79 \%\right.$ yield). ${ }^{1} \mathrm{H}$ NMR (600 $\left.\mathrm{MHz}, \mathrm{CD}_{2} \mathrm{Cl}_{2}\right): \delta 4.78(\mathrm{t}, J=1.8 \mathrm{~Hz}, 1 \mathrm{H}), 4.71(\mathrm{~s}, 1 \mathrm{H}), 2.38(\mathrm{~d}, J=17.4 \mathrm{~Hz}, 1 \mathrm{H}), 2.28-2.24$ (m, 2H), 2.09 (d, $J=17.4 \mathrm{~Hz}, 1 \mathrm{H}), 2.07-2.02(\mathrm{~m}, 1 \mathrm{H}), 1.88-1.84(\mathrm{~m}, 1 \mathrm{H}), 1.79(\mathrm{~s}, 3 \mathrm{H}), 1.17$ (s, 3H). ${ }^{13} \mathrm{C}$ NMR $\left(150 \mathrm{MHz}, \mathrm{CD}_{2} \mathrm{Cl}_{2}\right)$ : $\delta 218.9,151.6,109.5,51.6,45.3,37.1,34.3,25.9$, 19.8. HRMS (EI+): calcd for $\mathrm{C}_{9} \mathrm{H}_{14} \mathrm{O} 138.10447$, found 138.10502 .

3-Cyclopropylidene-1-methyl-1-(prop-1-en-2-yl)cyclopentane (3a)-Synthesized following general procedure B using 3-methyl-3-(prop-1-en-2-yl)cyclopentanone. Stirred 5 $\mathrm{h}$ at $62{ }^{\circ} \mathrm{C}$ after addition of ketone in THF. Purified by silica gel chromatography (hexanes) to give a colorless oil $\left(0.096 \mathrm{~g}, 18 \%\right.$ yield). ${ }^{1} \mathrm{H}$ NMR $\left(600 \mathrm{MHz}, \mathrm{CDCl}_{3}\right): \delta 4.70(\mathrm{~s}, 1 \mathrm{H}), 4.68$ (s, 1H), 2.47-2.41 (m, 3H), $2.23(\mathrm{~d}, J=15.0 \mathrm{~Hz}, 1 \mathrm{H}), 1.82-1.77(\mathrm{~m}, 1 \mathrm{H}), 1.76(\mathrm{~s}, 3 \mathrm{H}), 1.63-$ $1.58(\mathrm{~m}, 1 \mathrm{H}), 1.04(\mathrm{~s}, 3 \mathrm{H}), 0.97-0.95(\mathrm{~m}, 4 \mathrm{H}) .{ }^{13} \mathrm{C} \mathrm{NMR}\left(150 \mathrm{MHz}, \mathrm{CDCl}_{3}\right): \delta 153.1$, 
130.6, 111.4, 108.2, 47.7, 44.7, 37.2, 30.0, 25.4, 20.3, 2.5, 2.4. HRMS (EI+): calcd for $\mathrm{C}_{12} \mathrm{H}_{19}(\mathrm{M}+1) 163.14868$, found 163.14901 .

3-Methyl-3-(1-phenylvinyl)cyclopentanone-Synthesized following general procedure $\mathbf{C}$ using 3-methylcyclopent-2-enone. Stirred $4 \mathrm{~h}$ at $-41^{\circ} \mathrm{C}$ after addition of enone. Puri3cation by gradient silica gel chromatography (petroleum ether to 95:5 petroleum ether/ $\mathrm{Et}_{2} \mathrm{O}$ to $90: 5: 5$ petroleum ether/Et ${ }_{2} \mathrm{O} / \mathrm{EtOAc}$ ) provided the product compound as a yellow oil $\left(0.327 \mathrm{~g}, 18 \%\right.$ yield). ${ }^{1} \mathrm{H}$ NMR $\left(600 \mathrm{MHz}, \mathrm{CDCl}_{3}\right): \delta 7.31-7.26(\mathrm{~m}, 3 \mathrm{H}), 7.15-7.14(\mathrm{~m}, 2 \mathrm{H})$, $5.17(\mathrm{~s}, 1 \mathrm{H}), 4.96(\mathrm{~s}, 1 \mathrm{H}), 2.50(\mathrm{~d}, J=17.4 \mathrm{~Hz}, 1 \mathrm{H}), 2.31-2.28(\mathrm{~m}, 2 \mathrm{H}), 2.20-2.14(\mathrm{~m}, 1 \mathrm{H})$, $2.15(\mathrm{dd}, J=17.4,1.8 \mathrm{~Hz}, 1 \mathrm{H}), 1.86(\mathrm{ddt}, J=9.6,6.6,1.8 \mathrm{~Hz}, 1 \mathrm{H}), 1.25(\mathrm{~s}, 3 \mathrm{H}) .{ }^{13} \mathrm{C}$ NMR $\left(150 \mathrm{MHz}, \mathrm{CDCl}_{3}\right): \delta 218.8,156.4,142.3,128.8,128.0,127.2,113.8,51.9,45.2,36.7,34.5$, 26.6. HRMS (EI+): calcd for $\mathrm{C}_{14} \mathrm{H}_{16} \mathrm{O} 200.12012$, found 200.11936 .

(1-(3-Cyclopropylidene-1-methylcyclopentyl)vinyl)benzene (3b)-Synthesized following general procedure B using 3-methyl-3-(1-phenylvinyl)cyclopentanone. Stirred 8.5 $\mathrm{h}$ at $62{ }^{\circ} \mathrm{C}$ after addition of ketone in THF. Purified by silica gel chromatography (hexanes) to give a colorless oil $\left(0.221 \mathrm{~g}, 30 \%\right.$ yield). ${ }^{1} \mathrm{H}$ NMR $\left(600 \mathrm{MHz}, \mathrm{CDCl}_{3}\right): \delta 7.29-7.24(\mathrm{~m}$, $3 \mathrm{H}), 7.20-7.18(\mathrm{~m}, 2 \mathrm{H}), 5.17(\mathrm{~d}, J=1.2 \mathrm{~Hz}, 1 \mathrm{H}), 4.87(\mathrm{~d}, J=1.2 \mathrm{~Hz}, 1 \mathrm{H}), 2.57(\mathrm{~d}, J=15.6$ $\mathrm{Hz}, 1 \mathrm{H}), 2.44(\mathrm{bs}, 2 \mathrm{H}), 2.26(\mathrm{~d}, J=15.6 \mathrm{~Hz}, 1 \mathrm{H}), 1.97-1.92(\mathrm{~m}, 1 \mathrm{H}), 1.66-1.62(\mathrm{~m}, 1 \mathrm{H})$, $1.13(\mathrm{~s}, 3 \mathrm{H}), 0.96-0.94(\mathrm{~m}, 4 \mathrm{H}) .{ }^{13} \mathrm{C}$ NMR $\left(150 \mathrm{MHz}, \mathrm{CDCl}_{3}\right)$ : $\delta 158.4,143.7,130.3,128.8$, 127.7, 126.7, 112.6, 111.7, 47.8, 45.4, 37.8, 29.8, 26.2, 2.5, 2.4. HRMS (EI+): calcd for $\mathrm{C}_{17} \mathrm{H}_{20} 224.15650$, found 224.15584 .

3-(1-Phenylvinyl)cycloheptanone-Synthesized following general procedure $\mathbf{C}$ using 2-cyclohepten-1-one. Stirred $1.5 \mathrm{~h}$ at $-41{ }^{\circ} \mathrm{C}$ after addition of enone. Purification by gradient silica gel chromatography (petroleum ether to $95: 5$ petroleum ether/ $\mathrm{Et}_{2} \mathrm{O}$ to $90: 5: 5$ petroleum ether $/ \mathrm{Et}_{2} \mathrm{O} / \mathrm{EtOAc}$ ) provided the product compound as a pale yellow oil ( $1.13 \mathrm{~g}$, $58 \%$ yield). ${ }^{1} \mathrm{H}$ NMR $\left(400 \mathrm{MHz}, \mathrm{CDCl}_{3}\right): \delta 7.32-7.31(\mathrm{~m}, 4 \mathrm{H}), 7.29-7.26(\mathrm{~m}, 1 \mathrm{H}), 5.19(\mathrm{~s}$, $1 \mathrm{H}), 5.02(\mathrm{~s}, 1 \mathrm{H}), 2.82(\mathrm{t}, J=10.4 \mathrm{~Hz}, 1 \mathrm{H}), 2.70-2.61(\mathrm{~m}, 1 \mathrm{H}), 2.69(\mathrm{t}, J=14.4 \mathrm{~Hz}, 1 \mathrm{H})$, 2.55-2.51 (m, 2H), 2.07-2.03 (m, 1H), 1.99-1.90 (m, 2H), 1.69-1.59 (m, 1H), 1.52-1.35 $(\mathrm{m}, 2 \mathrm{H}) .{ }^{13} \mathrm{C} \mathrm{NMR}\left(100 \mathrm{MHz}, \mathrm{CDCl}_{3}\right): \delta 214.1,154.0,142.0,128.6,127.7,126.8,111.7$, 49.7, 44.2, 41.0, 37.3, 29.3, 24.4. HRMS (EI+): calcd for $\mathrm{C}_{15} \mathrm{H}_{18} \mathrm{O} 214.13577$, found 214.13519.

1-Cyclopropylidene-3-(1-phenylvinyl)cycloheptane (4a)-Synthesized following general procedure B using 3-(1-phenylvinyl)-cycloheptanone. Stirred $3.5 \mathrm{~h}$ at $62{ }^{\circ} \mathrm{C}$ after addition of ketone in THF. Purified by silica gel chromatography (hexanes) to give a colorless oil $\left(0.367 \mathrm{~g}, 47 \%\right.$ yield). ${ }^{1} \mathrm{H}$ NMR $\left(400 \mathrm{MHz}, \mathrm{CDCl}_{3}\right): \delta 7.39-7.37(\mathrm{~m}, 2 \mathrm{H}), 7.33-$ $7.29(\mathrm{~m}, 2 \mathrm{H}), 7.27-7.25(\mathrm{~m}, 1 \mathrm{H}), 5.19(\mathrm{~s}, 1 \mathrm{H}), 5.06(\mathrm{~s}, 1 \mathrm{H}), 2.71(\mathrm{~d}, J=14.0 \mathrm{~Hz}, 1 \mathrm{H}), 2.64$ $(\mathrm{t}, J=10.4 \mathrm{~Hz}, 1 \mathrm{H}), 2.52(\mathrm{~d}, J=14.8 \mathrm{~Hz}, 1 \mathrm{H}), 2.42-2.36(\mathrm{~m}, 1 \mathrm{H}), 2.28(\mathrm{t}, J=12.4 \mathrm{~Hz}, 1 \mathrm{H})$, 1.94-1.91 (m, 1H), 1.85-1.79 (m, 2H), 1.56-1.49 (m, 1H), 1.44-1.30 (m, 2H), 0.99-0.93 (m, 4H). ${ }^{13} \mathrm{C} \mathrm{NMR}\left(100 \mathrm{MHz}, \mathrm{CDCl}_{3}\right): \delta 155.7,143.0,128.9,128.4,127.3,126.8,116.8$, 110.6, 44.9, 41.0, 36.7, 34.6, 28.5, 27.8, 2.35. HRMS (EI+): calcd for $\mathrm{C}_{18} \mathrm{H}_{22}$ 238.17215, found 238.17115 .

6,7-Dimethyltricyclo[5.3.1.0]undec-4-ene (2a)—Colorless oil (0.019 g, 76\%). ${ }^{1} \mathrm{H}$ NMR (600 MHz, $\left.\mathrm{CDCl}_{3}\right): \delta 5.17(\mathrm{~s}, 1 \mathrm{H}), 2.82-2.75(\mathrm{~m}, 1 \mathrm{H}), 2.50-2.46(\mathrm{~m}, 1 \mathrm{H}), 2.01(\mathrm{bs}$, $1 \mathrm{H}), 1.74-1.70(\mathrm{~m}, 3 \mathrm{H}), 1.63(\mathrm{dd}, J=13.8,2.4 \mathrm{~Hz}, 1 \mathrm{H}), 1.50-1.40(\mathrm{~m}, 3 \mathrm{H}), 1.32(\mathrm{dt}, J=$ $12.3,4.2 \mathrm{~Hz}, 1 \mathrm{H}), 1.26(\mathrm{~d}, J=11.4 \mathrm{~Hz}, 1 \mathrm{H}), 0.94(\mathrm{~d}, J=7.2 \mathrm{~Hz}, 3 \mathrm{H}), 0.87(\mathrm{dt}, J=13.8,5.4$ $\mathrm{Hz}, 1 \mathrm{H}), 0.84$ (s, $3 \mathrm{H}) .{ }^{13} \mathrm{C}$ NMR $\left(150 \mathrm{MHz}, \mathrm{CDCl}_{3}\right): \delta 143.1,121.7,48.9,46.5,42.2,35.8$, $34.9,33.4,32.5,30.8,28.9,22.2,14.7$. HRMS (EI+): calcd for $\mathrm{C}_{13} \mathrm{H}_{20} 176.15650$, found 176.15578 . 
7-Methyl-6-phenyltricyclo[5.3.1.0]undec-4-ene (2b)-Synthesized following general procedure $\mathbf{D}$ using compound $\mathbf{1 b}$. Purified by silica gel chromatography (hexanes) to give a colorless oil ( $0.022 \mathrm{~g}, 88 \%$ yield). ${ }^{1} \mathrm{H}$ NMR $\left(600 \mathrm{MHz}, \mathrm{CDCl}_{3}\right): \delta 7.31-7.26(\mathrm{~m}, 4 \mathrm{H}), 7.22$ $7.20(\mathrm{~m}, 1 \mathrm{H}), 5.37(\mathrm{~s}, 1 \mathrm{H}), 3.35(\mathrm{~s}, 1 \mathrm{H}), 2.95-2.88(\mathrm{~m}, 1 \mathrm{H}), 2.59(\mathrm{dt}, J=13.8,5.4 \mathrm{~Hz}, 1 \mathrm{H})$, $1.91-1.88(\mathrm{~m}, 1 \mathrm{H}), 1.84-1.76(\mathrm{~m}, 3 \mathrm{H}), 1.70(\mathrm{~d}, J=10.8 \mathrm{~Hz}, 1 \mathrm{H}), 1.45-1.41(\mathrm{~m}, 3 \mathrm{H}), 1.07-$ $1.04(\mathrm{~m}, 1 \mathrm{H}), 0.97(\mathrm{~s}, 3 \mathrm{H}), 0.79(\mathrm{dt}, J=13.85 .4 \mathrm{~Hz}, 1 \mathrm{H}) .{ }^{13} \mathrm{C} \mathrm{NMR}\left(150 \mathrm{MHz}, \mathrm{CDCl}_{3}\right): \delta$ 145.2, 142.7, 130.0, 127.8, 126.2, 119.4, 55.4, 49.7, 46.4, 36.3, 35.4, 33.7, 32.6, 31.4, 29.3, 22.5. HRMS (EI+): calcd for $\mathrm{C}_{18} \mathrm{H}_{22} 238.17215$, found 238.17229.

\section{Supplementary Material}

Refer to Web version on PubMed Central for supplementary material.

\section{Acknowledgments}

The UNC group acknowledges the National Institute of General Medicine (GM-60578). The UCD group acknowledges the National Science Foundation, the University of California Institute for Mexico and the United States, UCD \& Humanities Graduate Research Award, and an R. B. Miller Graduate Fellowship to O.G.

\section{References}

1. Wendt KU, Schulz GE, Corey EJ, Liu DR. Angew Chem, Int Ed. 2000; 39:2812.

2. Yoder RA, Johnston JN. Chem Rev. 2005; 105:4730. [PubMed: 16351060]

3. Rubin M, Rubina M, Gevorgyan V. Chem Rev. 2007; 107:3117. [PubMed: 17622181]

4. Reissig HU, Zimmer R. Chem Rev. 2003; 103:1151. [PubMed: 12683780]

5. Duffy RJ, Morris KA, Romo D. Tetrahedron. 2009; 65:5879. [PubMed: 20640036]

6. Wolk JL, Rozental E, Basch H, Hoz S. J Org Chem. 2006; 71:3876. [PubMed: 16674063]

7. Seiser T, Cramer N. Org Biomol Chem. 2009; 7:2835. [PubMed: 19582290]

8. Murakami M, Ashida S, Matsuda T. J Am Chem Soc. 2006; 128:2166. [PubMed: 16478142]

9. Kondo T, Nakamura A, Okada T, Suzuki N, Wada K, Mitsudo T. J Am Chem Soc. 2000; 122:6319. 10. Fürstner A, Aïssa C. J Am Chem Soc. 2006; 128:6306. [PubMed: 16683781]

11. Seiser T, Saget T, Tran DN, Cramer N. Angew Chem, Int Ed. 2011; 50:7740.

12. Kotora M, Hessler F, Eignerová B. Eur J Org Chem. 2012:29.

13. Moyano A, Rios R. Chem Rev. 2011; 111:4703. [PubMed: 21615119]

14. Chen DYK, Youn SW. Chem—Eur J. 2012; 18:9452. [PubMed: 22736530]

15. Razzak M, Brabander JKD. Nat Chem Biol. 2012; 7:865. [PubMed: 22086288]

16. Enders D, Narine AA. J Org Chem. 2008; 73:7857. [PubMed: 18778100]

17. Felix RJ, Weber D, Gutierrez O, Tantillo DJ, Gagné MR. Nature Chem. 2012; 4:405. [PubMed: 22522261]

18. Johnson WTG, Borden WT. J Am Chem Soc. 1997; 119:5930.

19. Bach RD, Dmitrenko O. J Am Chem Soc. 2004; 126:4444. [PubMed: 15053635]

20. Majumdar KC, Chattopadhyay B. Curr Org Chem. 2009; 13:731.

21. Prunet J. Eur J Org Chem. 2011; 35:3635.

22. Watson IDG, Ritter S, Toste FD. J Am Chem Soc. 2009; 131:2056. [PubMed: 19161306]

23. Siengalewicz P, Mulzer J, Rinner U. Eur J Org Chem. 2011; 35:7041.

24. El-Hachach N, Gerke R, Noltemeyer M, Fitjer L. Tetrahedron. 2009; 65:1040.

25. Zu L, Xu M, Lodewyk MW, Cane DE, Peters RJ, Tantillo DJ. J Am Chem Soc. 2012; 134:1136911371. [PubMed: 22738258]

26. Abraham WR. Curr Med Chem. 2001; 8:583. [PubMed: 11281843]

27. Mézailles N, Ricard L, Gagosz F. Org Lett. 2005; 7:4133. [PubMed: 16146370] 
28. Model made in PyMOL from the lowest energy conformer found in a conformer distribution calculation performed in MAC Spartan '10 (Wavefunction Inc.) at the B3LYP 6-31G(d) level of theory. See: Becke AD. J Chem Phys. 1993; 98:1372.

29. Becke AD. J Chem Phys. 1993; 98:1372.

30. Lee C, Yang W, Parr RG. Phys Rev B. 1988; 37:785.

31. Stephens PJ, Devlin FJ, Chabalowski CF, Frisch MJ. J Phys Chem. 1994; 98:11623.

32. Tirado-Rives J, Jorgensen WL. J Chem Theory Comput. 2008; 4:297.

33. Stafford JA, McMurry JE. Tetrahedron Lett. 1988; 29:2531.

34. Frisch, MJ., et al. GAUSSIAN09, Revision A. 02. Gaussian Inc; Wallingford, CT: 2009.

35. Zhao Y, Truhlar DG. Theor Chem Acc. 2008; 120:215.

36. Zhao Y, Truhlar DG. Acc Chem Res. 2008; 41:157. [PubMed: 18186612]

37. Cossi M, Rega N, Scalmani G, Barone VJ. Comput Chem. 2003; 24:669.

38. Although the free energy of the transition state structure A3-TS is lower than A4, the electronic energy of intermediate $\mathbf{A 4}$ is $0.3 \mathrm{kcal} / \mathrm{mol}$ lower than A3-TS.

39. Müller D, Tissot M, Alexakis A. Org Lett. 2011; 13:3040. [PubMed: 21591618] 

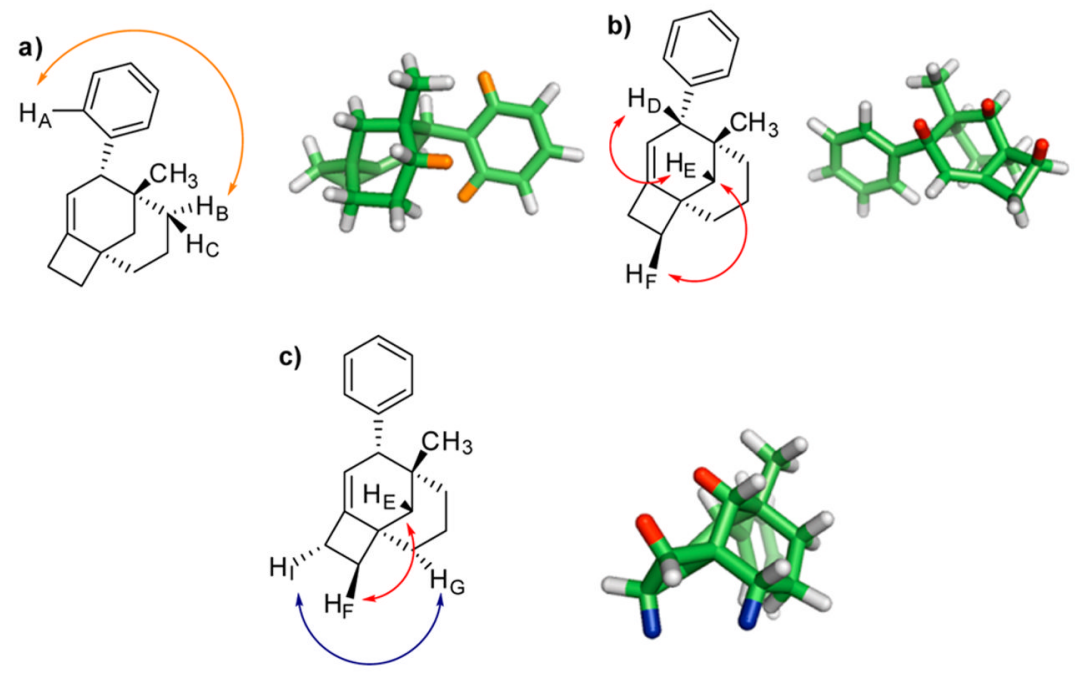

Figure 1.

Selected NOESY correlations in NMR spectrum of bicyclo[4.2.0]oct-1-ene 2b. 3D representations made in PyMOL. ${ }^{28-32}$ 

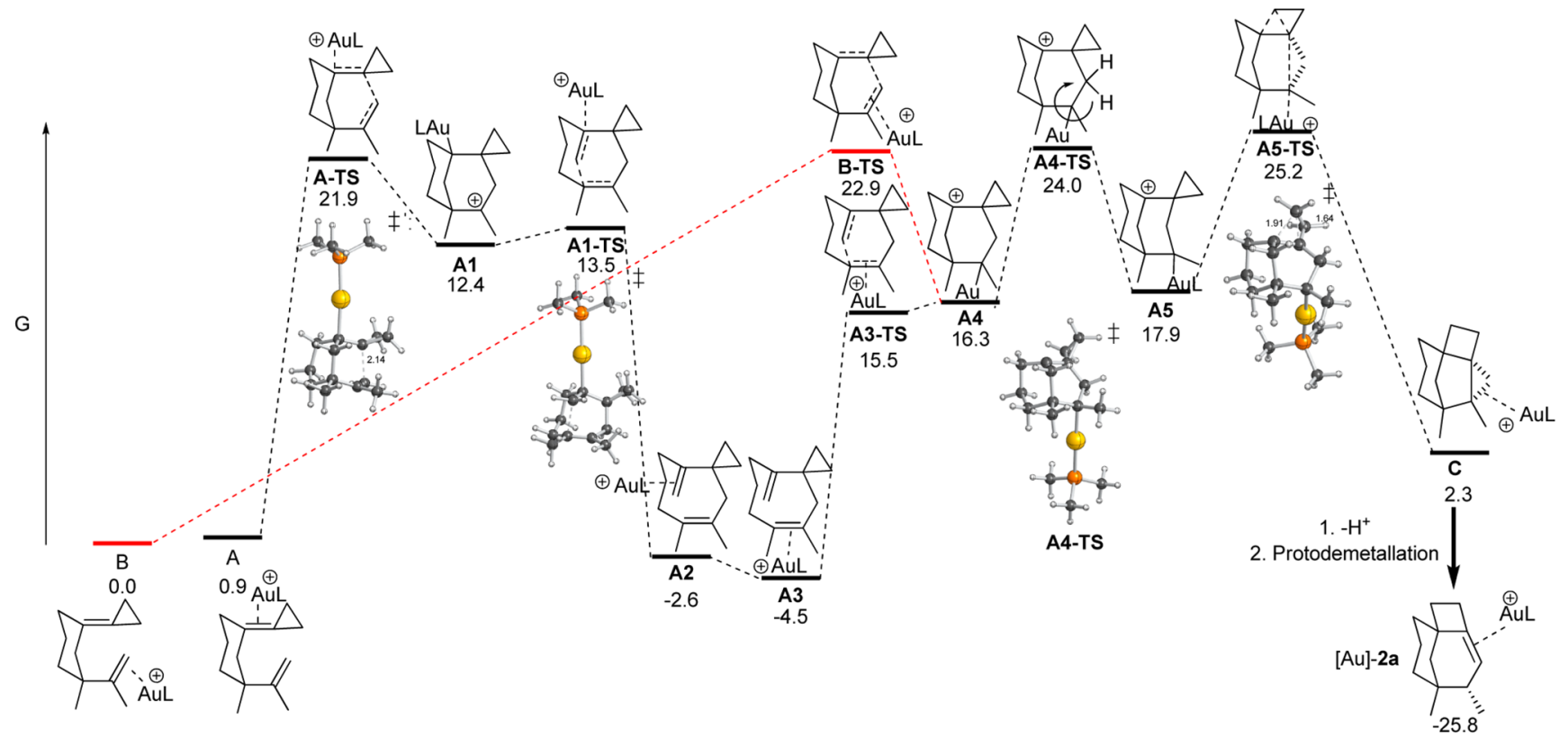

Figure 2.

Computed relative free energies $(\mathrm{kcal} / \mathrm{mol})$ for species involved in potential rearrangements of 1a. 
Au-Catalyzed Cope Rearrangement of Acyclic Substrates<smiles>C=CC1([C@@H](CCCC=C(C)C)C(=C)C)CC1</smiles>

Proposed Cope Rearrangement of Cyclic Substrates

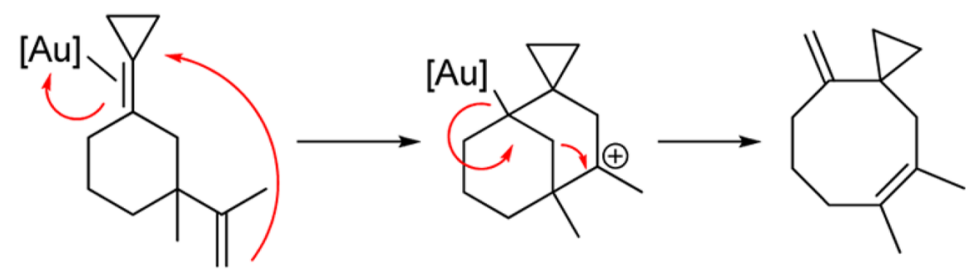

Scheme 1.

Gold-Catalyzed Cope Rearrangements 
<smiles>[R]C=[R]C=[R]</smiles>

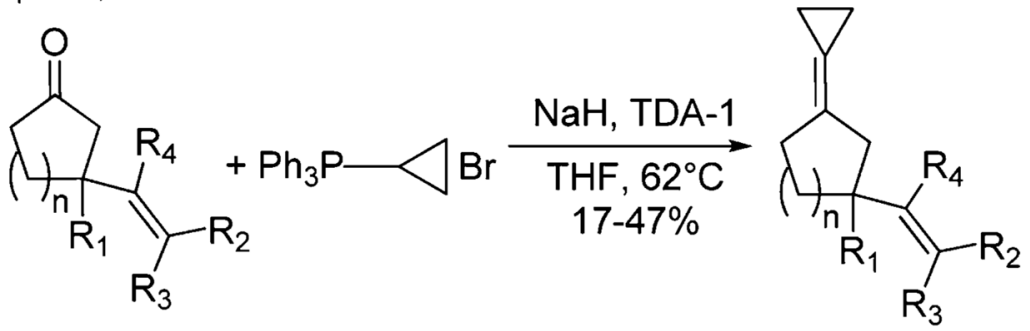<smiles>CC=CC1(C)CCCC(=C2CC2)C1</smiles>

1c

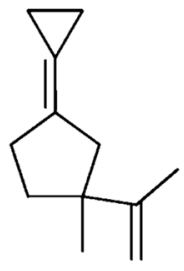

$3 a$<smiles>CC=C(C)C1(C)CCCC(=C2CC2)C1</smiles>

1d<smiles>C=C(c1ccccc1)C1(C)CCC(=C2CC2)C1</smiles>

3b<smiles>CC(C)=CC1(C)CCCC(=C2CC2)C1</smiles>

$1 \mathrm{e}$

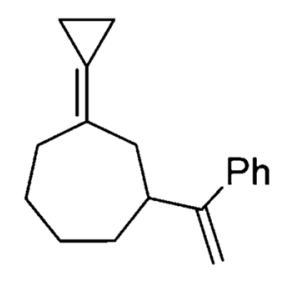

$4 a$

Scheme 2.

Synthesis of Cyclic Substrates 


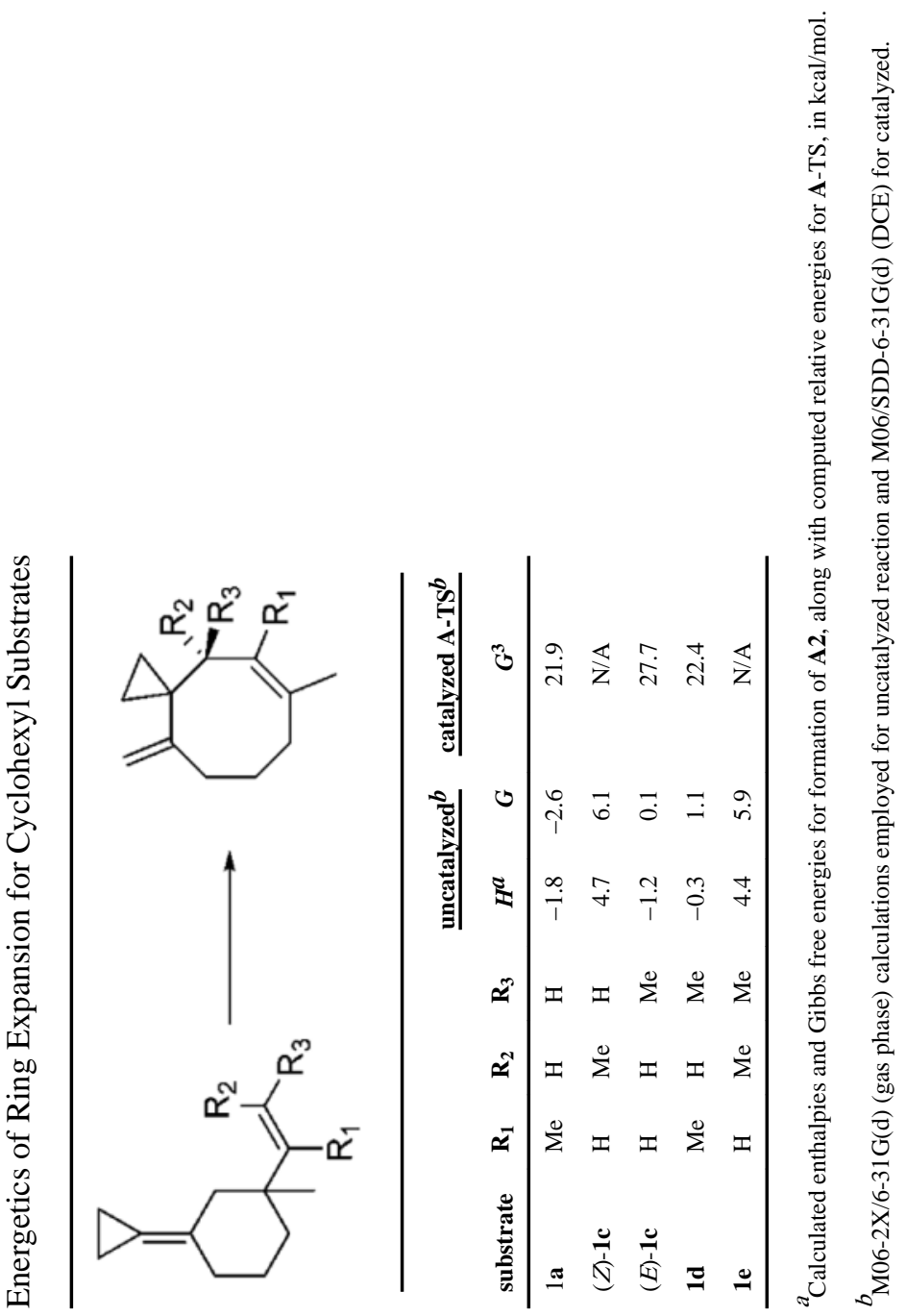

J Org Chem. Author manuscript; available in PMC 2013 October 02. 